\title{
EFEITO DA IDADE E CLONE NA QUALIDADE DA MADEIRA DE Eucalyptus spp VISANDO À PRODUÇÃO DE BIOENERGIA
}

\author{
EFFECT OF AGE AND CLONE ON THE QUALITY OF Eucalyptus spp WOOD AIMING \\ BIOENERGY PRODUCTION
}

\author{
Thiago de Paula Protásio ${ }^{1}$ Thiago Andrade Neves ${ }^{2}$ Aliny Aparecida dos Reis ${ }^{3}$ \\ Paulo Fernando Trugilho ${ }^{4}$
}

\begin{abstract}
RESUMO
O objetivo deste trabalho foi avaliar o efeito da idade e clone na qualidade da madeira de Eucalyptus spp visando à produção de carvão vegetal e bioenergia. Foram avaliados quatro clones de Eucalyptus spp nas idades de 57 e 69 meses. Foram amostradas quatro árvores por clone em cada idade. Determinou-se a densidade básica média, a densidade básica no DAP, o poder calorífico superior e os teores de lignina, extrativos totais, cinzas, holocelulose e a análise química elementar $(\mathrm{C}, \mathrm{H}, \mathrm{N}, \mathrm{S}$ e $\mathrm{O})$. Na avaliação do experimento utilizou-se um delineamento inteiramente casualizado em um esquema fatorial $4 \times 2$. Além disso, foi realizada a análise multivariada de componentes principais. Observou-se, de maneira geral, efeito da idade na qualidade da madeira. Os teores de cinzas, hidrogênio e nitrogênio tenderam a diminuir com o aumento da idade para todos os clones avaliados. Já o teor de oxigênio apresentou um acréscimo com a idade dos clones. $\mathrm{O}$ teor de lignina apresentou significativo aumento com a idade, sendo o contrário observado para o teor de holocelulose. Os clones de 69 meses apresentaram os maiores teores de lignina, mas não os maiores valores para a densidade básica da madeira. Já os clones I144 e I220 apresentaram os maiores valores médios para essa característica, o que pode ser benéfico para a produção de carvão vegetal. Palavras-chave: biomassa; bioenergia; carvão vegetal; composição química.
\end{abstract}

\begin{abstract}
The aim of this work was to evaluate the effect of age and clone on the quality of Eucalyptus spp wood aiming the production of charcoal and bioenergy. Four Eucalyptus spp clones at the ages of 57 and 69 months were evaluated. Four trees per clone in each age were evaluated. The average basic density, the basic density at the $\mathrm{DBH}$, higher heating value and contents of lignin, total extractives, ashes, holocellulose an elemental chemica analylsis $(\mathrm{C}, \mathrm{H}, \mathrm{N}, \mathrm{S}$ and $\mathrm{O})$ were evaluated. In the evaluation of the assay, and entirely randomized design in the factorial scheme $4 \times 2$ was used. Besides, a multivariate analysis of main components was made. In general, it was observed effect of age on wood quality. Ash, hydrogen and nitrogen contents tended to diminish with increases in age for all the clones evaluated. However, oxygen content presented an increase with the increase in age of the clones. Lignin content presented significant increase with age increase, being the contrary observed for holocellulose content. The clones at the age of 69 months presented the highest contents of lignin, but the same did not happen for basic density of wood. The clones I144 and I220 had the highest average values for this characteristic, what may be advantageous
\end{abstract}

1 Engenheiro Florestal, Doutorando em Ciência e Tecnologia da Madeira, Departamento de Ciências Florestais, Universidade Federal de Lavras, Campus Universitário, Caixa Postal 3037, CEP 37200-000, Lavras (MG), Brasil. depaulaprotasio@gmail.com

2 Engenheiro Florestal, Msc., Saint-Gobain Pam Bioenergia Ltda., Rua Corrêa Lacerda, 33, CEP 37310-000, Bom Jardim (MG), Brasil. thiago.neves@saint-gobain.com

3 Engenheira Florestal, Mestranda em Engenharia Florestal, Universidade Federal de Lavras, Câmpus Universitário, Caixa Postal 3037, CEP 37200-000, Lavras (MG), Brasil, alinyreis@hotmail.com

4 Engenheiro Florestal, Dr., Professor do Departamento de Ciências Florestais, Universidade Federal de Lavras, Câmpus Universitário, Caixa Postal 3037, CEP 37200-000, Lavras (MG), Brasil. trugilho@dcf.ufla.br

Recebido para publicação em 7/11/2011 e aceito em 14/12/2012

Ci. Fl., v. 24, n. 2, abr.-jun., 2014 
for charcoal production.

Keywords: biomass; bioenergy; charcoal; chemical composition.

\section{INTRODUÇÃO}

Nas últimas décadas e, principalmente nos últimos anos, o Brasil apresentou um crescimento econômico considerável e acima de vários países do mundo. Esse crescimento foi acompanhado de um aumento na demanda de energia do país. Segundo dados da Empresa de Pesquisa Energética (EPE, 2011), do Ministério de Minas e Energia, o país consumiu 419.016 GWh de energia elétrica em 2010 e, com o aumento do PIB (Produto Interno Bruto), essa demanda por energia deve crescer cerca de $74 \%$ em 2020. Portanto, a busca por desenvolvimento de tecnologias na área energética vem apresentando caráter fundamental para o desenvolvimento do país nessa década.

Novas pesquisas científicas relacionadas à potencialidade de implementação das energias alternativas estão sendo desenvolvidas, em virtude de essas serem de origem renovável e por possuírem baixo potencial poluidor do meio ambiente. Atualmente, a maioria dos países visa ao aumento da participação da bioenergia nas suas matrizes energéticas e, consequentemente, diminuição das emissões de dióxido de carbono na atmosfera advindo principalmente da queima de combustíveis fósseis (não renováveis).

No Brasil, há uma crescente demanda pela utilização da biomassa para uso industrial, principalmente para o abastecimento de caldeiras e para a geração de energia na forma direta ou para a redução do minério de ferro. Cerca de $18,4 \%$ dos plantios de eucaliptos são destinados à produção de carvão vegetal para o abastecimento dos fornos siderúrgicos, sendo este setor o maior consumidor e produtor deste insumo. A área de plantios de Eucalyptus spp no Brasil é de 4.873.952 hectares e continua em processo de expansão. O estado de Minas Gerais apresenta a maior área plantada (aproximadamente $30 \%$ da área cultivada), destinada, principalmente, para atender ao maior parque siderúrgico a carvão vegetal do Brasil instalado nesse estado. Contudo, atualmente, $55 \%$ da produção brasileira de carvão vegetal são advindas de florestas nativas e a tendência é que essa percentagem diminua a cada ano. O Brasil se destaca no cenário bioenergético por ser o maior produtor de carvão vegetal do mundo (ABRAF, 2012).

Uma das principais fontes de energia de biomassa utilizadas é o carvão vegetal, que, quando comparado ao carvão mineral, apresenta inúmeras vantagens por oferecer em sua constituição química, baixos teores de minerais, sendo praticamente isento de enxofre e fósforo (NEVES et al., 2011; ABRAF, 2012; ASSIS et al., 2012; REIS et al., 2012). Além disso, o carvão vegetal é um agente de dupla finalidade, ou seja, gera energia (calor) para o sistema e reduz o minério de ferro (PINHEIRO et al., 2006).

Entretanto, o carvão vegetal é altamente variável em qualidade, uma vez que a madeira e os processos utilizados na pirólise influenciam diretamente a qualidade do carvão vegetal, sendo que a homogeneidade desse biocombustível é fundamental para o processo de redução do minério de ferro e produção de aço e ferro-gusa de boa qualidade. Dessa forma, pesquisas relacionadas à avaliação de clones de Eucalyptus spp para a produção de bioenergia e carvão vegetal para a siderurgia são de grande importância.

O potencial energético de uma espécie sofre influência de diversos fatores, tais como a sua constituição genética, tratamentos silviculturais, idade, local de plantio e interações, podendo afetar de forma considerável a qualidade da madeira e, consequentemente, do carvão vegetal. Nesse contexto, tornam-se necessárias a avaliação e quantificação de inúmeras características na madeira que influenciam direta ou indiretamente na qualidade e na produção do carvão vegetal, como os teores de lignina, extrativos totais, holocelulose e cinzas, além da densidade básica (TRUGILHO et al., 1997; TRUGILHO, 2009; TRUGILHO et al., 2001; VALE et al., 2010; PROTÁSIO et al., 2012). Além disso, a quantificação dos teores dos componentes elementares da madeira, ou seja, carbono, hidrogênio, oxigênio, enxofre e nitrogênio também são importantes, pois está relacionada ao valor calórico e ao desempenho energético de combustíveis de biomassa vegetal (DEMIRBAS, 2004; OBERNBERGER et al., 2006; HUANG et al., 2009; PAULA et al., 2011; PROTÁSIO et al., 2011). 
Nesse sentido, a busca por materiais genéticos superiores quanto às características favoráveis à produção de carvão vegetal é de suma importância no aumento do rendimento do processo de carbonização, bem como na melhoria da qualidade do carvão vegetal produzido.

Sendo assim, o objetivo deste trabalho foi avaliar o efeito da idade e clone na qualidade da madeira de Eucalyptus spp visando à produção de carvão vegetal e bioenergia.

\section{MATERIAL E MÉTODOS}

\section{Material biológico e amostragem}

Foram amostrados quatro clones de Eucalyptus spp da empresa Saint Gobain Pam Bioenergia, localizada no município de Bom Jardim de Minas (Minas Gerais - Brasil). A região de coleta apresenta temperatura e precipitação média de $20,4^{\circ} \mathrm{C}$ e $1.250 \mathrm{~mm}$, respectivamente. Os clones apresentavam idade de 57 e 69 meses e o espaçamento de plantio foi de $3,5 \times 2,5 \mathrm{~m}$ (Tabela 1). Os clones amostrados I144 e I220 são provenientes da empresa Acesita Energética e os clones 3334 e 3281 da empresa Plantar.

TABELA 1: Plano experimental.

TABLE 1: Experimental plan.

\begin{tabular}{cccccc}
\hline Tratamento & Clone & $\begin{array}{c}\text { Idade } \\
\text { (meses) }\end{array}$ & $\begin{array}{c}\text { DAP } \\
(\mathrm{cm})\end{array}$ & HT (m) & HC (m) \\
\hline T1 & I144 & 57 & 16,79 & 24,60 & 21,20 \\
T2 & I220 & 57 & 17,28 & 22,80 & 19,08 \\
T3 & 3281 & 57 & 20,89 & 24,07 & 20,90 \\
T4 & 3334 & 57 & 12,41 & 22,10 & 19,03 \\
T5 & I144 & 69 & 19,20 & 27,60 & 25,80 \\
T6 & I220 & 69 & 18,77 & 24,75 & 22,35 \\
T7 & 3281 & 69 & 19,38 & 27,10 & 25,43 \\
T8 & 3334 & 69 & 16,81 & 25,58 & 24,00 \\
\hline
\end{tabular}

Em que: DAP $=$ diâmetro a altura do peito; $\mathrm{HT}=$ altura total; $\mathrm{HC}=$ altura comercial.

Foram amostradas de forma casual quatro árvores de cada clone, sendo retirados discos de $2,5 \mathrm{~cm}$ de espessura a $2,10,30,50$ e $70 \%$ da altura comercial da árvore (considerada até um diâmetro mínimo de $5 \mathrm{~cm}$ com casca), além de um disco extra a 1,30 m de altura do solo (DAP), conforme diretrizes de Downes et al. (1997).

\section{Análises químicas, físicas e energéticas da madeira}

Os discos retirados das árvores foram seccionados em quatro cunhas passando pela medula. As duas menores cunhas opostas foram utilizadas na determinação da densidade básica da madeira, seguindo os preceitos da norma NBR 11941 (ABNT, 2003). A densidade básica média da árvore $(\mathrm{DBm})$ foi considerada como sendo a média aritmética dos pontos de amostragem longitudinal no tronco das árvores, sem considerar o DAP.

Para a determinação do poder calorífico superior (PCS), dos teores de lignina, extrativos totais, cinzas e dos componentes elementares (C, H, N, S e O) foram retiradas amostras (maravalhas) de todos os discos, por meio de uma plaina elétrica manual, visando à formação de uma amostra composta por árvore. Para a obtenção destas amostras foram utilizadas as cunhas não destinadas ao ensaio de densidade básica. Posteriormente, as amostras foram moídas em um moinho tipo Wiley e peneiradas.

$\mathrm{O}$ teor de lignina insolúvel (Klason) foi obtido de acordo com a metodologia proposta por Gomide e Demuner (1986) e o teor de lignina solúvel (Klason) em ácido sulfúrico foi determinado de acordo com a metodologia proposta por Goldschimid (1971). O teor de lignina total foi obtido pelo somatório dos teores de lignina solúvel e insolúvel.

Para a quantificação dos teores de cinzas e extrativos totais da madeira foram consideradas as metodologias previstas nas normas M11/77 e M3/69 (ABTCP, 1974a; 1974b), respectivamente. O teor de holocelulose foi obtido por diferença [Holocelulose $(\%)=100-$ Lignina total $(\%)-$ Extrativos totais $(\%)$ - Cinzas (\%)].

Para a análise elementar, as amostras compostas foram moídas e peneiradas, sendo utilizada a fração que passou pela peneira de 60 mesh e ficou retida pela peneira de 270 mesh. A quantificação dos teores de carbono, hidrogênio, nitrogênio e enxofre em relação à massa seca da madeira foi realizada em um analisador universal da marca Elementar (modelo Vario Micro Cube), conforme realizado por Neves et al. (2011), Paula et al. (2011), Protásio et al. (2011) e Reis et al, (2012). $\mathrm{O}$ analisador utiliza como gases de arraste e ignição o hélio e o oxigênio, respectivamente. As amostras de $2 \mathrm{mg}$ foram acondicionadas em cápsulas de estanho e completamente incineradas a $1.200^{\circ} \mathrm{C}$. O 
teor de oxigênio foi obtido por diferença em relação aos demais componentes elementares.

O poder calorífico superior foi determinado em um calorímetro digital IKA C-200, conforme a norma NBR 8633 (ABNT, 1984).

\section{Análise estatística univariada: fatorial duplo}

$\mathrm{Na}$ avaliação das características quantificadas na madeira utilizou-se o delineamento inteiramente casualizado disposto em um esquema fatorial $4 \times 2$ considerando-se como níveis dos fatores os diferentes clones e a idade e 4 repetições, conforme modelo estatístico apresentado na Eq. 1:

$$
\mathrm{Y}_{\mathrm{ijk}}=\mu+\tau_{\mathrm{i}}+\beta_{\mathrm{j}}+(\tau \beta)_{\mathrm{ij}}+\varepsilon_{\mathrm{ijk}}
$$

Onde: $Y_{\mathrm{ijk}}$ é a observação (características da madeira) do $i$-ésimo nível do fator clone ( $i=1,2,3$ e 4), no $j$-ésimo nível do fator idade $(\mathrm{j}=1$ e 2$)$ na $k$-ésima repetição $(\mathrm{k}=1,2,3$ e 4$)$; $\mu$ é uma constante inerente a todas as observações; $\mathrm{t}_{\mathrm{i}}$ é o efeito do $i$-ésimo nível do fator clone $(\mathrm{i}=1$, 2, 3 e 4), efeito fixo; $b_{j}$ é o efeito do $j$-ésimo nível do fator idade $(\mathrm{j}=1 \text { e 2), efeito fixo; (tb) })_{\mathrm{ij}}$ é o efeito da interação entre $t_{i}$ e $b_{j}$, efeito aleatório, e e $e_{i j k}$ é o erro experimental associado à observação $\mathrm{Y}_{\mathrm{i} \mathrm{j}}$, independente e identicamente distribuído de uma Normal com média zero e variância $\sigma^{2}$.

\section{Análise estatística multivariada: componentes} principais

Foram utilizadas apenas as médias de cada variável e a análise de componentes principais foi realizada considerando-se os dados padronizados, ou seja, com variância unitária.

Ferreira (2008) e Mingoti (2005) afirmam que as componentes principais obtidas via matriz de covariâncias são influenciadas pelas variáveis de maior variância ou pelo efeito da escala das variáveis, sendo que esse problema pode ser amenizado se as componentes forem obtidas via modelagem da matriz de correlação dos dados originais. Esse procedimento equivale a padronizar as variáveis e permite maior acurácia na análise (MINGOTI, 2005).

Todas as análises estatísticas foram efetuadas utilizando o software estatístico R versão 2.15.1 pacotes stats (R DEVELOPMENT CORE TEAM, 2012) e SensoMineR (HUSSON et al., 2012).

\section{RESULTADOS E DISCUSSÃO}

\section{Análise estatística univariada: fatorial duplo}

Observa-se que o efeito da interação entre clone $\mathrm{x}$ idade foi significativo para os teores de hidrogênio, nitrogênio, enxofre, oxigênio, extrativos totais e cinzas (Tabelas 2 e 3 ), evidenciando a existência de dependência entre os fatores considerados (clone e idade). Dessa forma, procedeu-se o desdobramento de clone dentro de idade e idade dentro de clone.

Para o teor de carbono observou-se efeito significativo somente para o fator idade (Tabela 2). Já para a densidade básica obtida no DAP e média observou-se efeito significativo apenas de clone. Para os teores de lignina e holocelulose observou-se efeito significativo da idade e clone (Tabela 3). Para essas variáveis procedeu-se a análise dos efeitos simples.

Não foram observados efeitos significativos de clone, idade ou da interação para o poder calorífico superior (Tabela 2).

Observa-se ainda que somente o coeficiente de variação do teor de enxofre apresentou valor elevado, fato este que pode ser explicado pelo baixo valor percentual deste constituinte químico na madeira.

Analisando o efeito da idade dentro de cada nível de clone para os teores de extrativos totais e enxofre (Figuras 1 e 2), observa-se que os clones 3334 e I144 não apresentaram diferença estatística nas duas idades avaliadas, sendo o contrário observado para os clones 3281 e I220. O clone I220 apresentou o maior valor médio do teor de extrativos totais aos 57 meses (Figura 1). No clone 3281 observa-se um aumento do teor de extrativos totais com o aumento na idade (Figura 1). Segundo Severo et al. (2006), há uma tendência do teor de extrativos totais ser menor em madeiras mais jovens devido à maior quantidade de madeira juvenil.

Considerando o teor de enxofre para os clones 3281 e I220, ambos apresentaram uma tendência de decréscimo do valor médio com o aumento da idade (Figura 2). Contudo, os valores encontrados foram baixos e não comprometem a utilização energética da biomassa avaliada. Para os clones 3281 e I144, na idade de 69 meses, não foi detectado enxofre na madeira, justificando assim a ausência de barras no gráfico (Figura 2). Esse resultado pode ser considerado uma vantagem, 
TABELA 2: Resumo das análises de variância realizadas para os teores dos componentes químicos elementares e para o poder calorífico superior da madeira dos clones de Eucalyptus spp.

TABLE 2: Summary of the variance analyses made for the chemical elemental components and for higher he ating value of Eucalyptus clones wood.

\begin{tabular}{cccccccc}
\hline \multirow{2}{*}{ Fator de variação } & \multirow{2}{*}{$\begin{array}{c}\text { Graus de } \\
\text { Liberdade }\end{array}$} & Carbono & Hidrogênio & Nitrogênio & Enxofre & Oxigênio & PCS \\
\cline { 3 - 7 } & 3 & $0,948^{\text {ns }}$ & $0,088^{\text {ns }}$ & $0,001^{\text {ns }}$ & $0,013^{*}$ & $1,662^{\text {ns }}$ & $6178^{\text {ns }}$ \\
Clone & 1 & $68,312^{*}$ & $9,143^{*}$ & $0,063^{*}$ & $0,058^{*}$ & $138,905^{*}$ & $2410^{\text {ns }}$ \\
Idade & 3 & $2,926^{\text {ns }}$ & $0,378^{*}$ & $0,009^{*}$ & $0,028^{*}$ & $6,169^{*}$ & $9854^{\text {ns }}$ \\
\hline Clone x Idade & 23 & 1,28950 & 0,03887 & 0,00071 & 0,00256 & 1,82276 & $10172,80^{\text {ns }}$ \\
\hline Erro & CVe $(\%)$ & 2,44 & 3,14 & 14,59 & 73,71 & 2,88 & 2,22 \\
\hline
\end{tabular}

Em que: $\mathrm{CVe}(\%)$ = coeficiente de variação experimental; $\mathrm{PCS}=$ poder calorífico superior; ${ }^{*}=$ significativo a $5 \%$ pelo Teste $\mathrm{F}{ }^{\text {ns }}=$ não significativo a $5 \%$ pelo Teste $\mathrm{F}$.

pois a liberação de enxofre na atmosfera está associada à formação de óxidos SOx e chuva ácida e, consequentemente, corrosão (BILGEN; KAYGUSUZ, 2008; BECH et al., 2009), além de prejudicar as propriedades do ferro-gusa (ASSIS et al., 2012). As diminutas concentrações de enxofre na madeira é um aspecto positivo que viabiliza e aumenta o uso de combustíveis de biomassa vegetal pelos diversos setores industriais.

Verificam-se para os teores de cinzas, hidrogênio e oxigênio (Figuras 3, 4 e 5) que todos os clones apresentaram médias estatisticamente diferentes nas duas idades avaliadas, ou seja, para essas características observou-se o significativo efeito da idade. Os teores de cinzas (Figura 3) e hidrogênio (Figura 4) decresceram com o aumento da idade, porém, o teor de oxigênio apresentou comportamento contrário para todos os clones.

Do ponto de vista bioenergético, os minerais presentes nos combustíveis de biomassa são prejudiciais, pois podem formar incrustações nos equipamentos e nas tubulações quando a biomassa é queimada em fornalhas, além de diminuir o poder calorífico do material combustível (BRAND, 2010; PAULA et al., 2011; PROTÁSIO et al., 2011; REIS et al., 2012). No processo de carbonização para uso siderúrgico, a madeira deve possuir baixo teor de minerais, uma vez que estes diminuem a qualidade do aço e das ligas metálicas produzidas (BARCELLOS et al., 2005). Logo, o decréscimo observado para o teor de cinzas com a idade é vantajoso para a geração de energia.

Quanto aos componentes elementares, um aumento do teor de oxigênio e diminuição do teor de hidrogênio tende a diminuir o poder calorífico de combustíveis de biomassa vegetal (PAULA et al., 2011; PROTÁSIO et al., 2011). Contudo, não foi observado efeito da idade, clone ou da interação

TABELA 3: Resumo das análises de variância para a densidade básica e os teores de extrativos totais, lignina, cinzas e holocelulose.

TABLE 3: Summary of the variance analyses for basic density and total extractives, lignin, ashes and holocellulose contents.

\begin{tabular}{|c|c|c|c|c|c|c|c|}
\hline \multirow{2}{*}{$\begin{array}{l}\text { Fator de } \\
\text { variação }\end{array}$} & \multirow{2}{*}{$\begin{array}{l}\text { Graus de } \\
\text { Liberdade }\end{array}$} & \multicolumn{6}{|c|}{ Quadrado Médio } \\
\hline & & $\mathrm{DB}_{\text {DAP }}$ & $\mathrm{DBm}$ & $\begin{array}{c}\text { Extrativos } \\
\text { totais }\end{array}$ & $\begin{array}{c}\text { Lignina } \\
\text { total }\end{array}$ & Cinzas & Holocelulose \\
\hline Clone & 3 & $0,0145003^{*}$ & $0,01304^{*}$ & $3,566^{*}$ & $8,07^{*}$ & $0,036^{*}$ & $18,550^{*}$ \\
\hline Idade & 1 & $0,0000001^{\mathrm{ns}}$ & $0,00012^{\mathrm{ns}}$ & $0,002^{\mathrm{ns}}$ & $184,59^{*}$ & $0,180^{*}$ & $174,570^{*}$ \\
\hline Clone $\mathrm{x}$ Idade & 3 & $0,0001718^{\mathrm{ns}}$ & $0,00009^{\mathrm{ns}}$ & $1,870^{*}$ & $0,810^{\text {ns }}$ & $0,006^{*}$ & $2,875^{\mathrm{ns}}$ \\
\hline \multirow[t]{2}{*}{ Erro } & 23 & 0,0002834 & 0,0002534 & 0,19277 & 1,656 & 0,00158 & 1,98521 \\
\hline & $\mathrm{CVe}(\%)$ & 3,68 & 3,46 & 14,23 & 4,56 & 13,5 & 2,06 \\
\hline
\end{tabular}

Em que: CVe (\%) = coeficiente de variação experimental; $\mathrm{DB}_{\mathrm{DAP}}$ : densidade básica no DAP; DBm: densidade básica média; ${ }^{*}=$ significativo a $5 \%$ pelo Teste $\mathrm{F} ;{ }^{\text {ns }}=$ não significativo a $5 \%$ pelo Teste $\mathrm{F}$. 


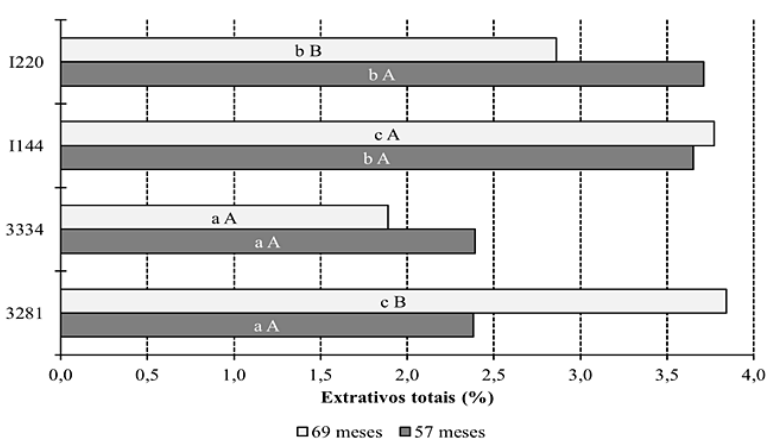

FIGURA 1: Teor de extrativos totais da madeira dos clones nas duas idades avaliadas. Barras seguidas de mesma letra minúscula referem-se ao desdobramento de clone dentro de idade. Já as letras maiúsculas referem-se ao desdobramento de idade dentro de clone. O teste utilizado foi o de Tukey $(\alpha=0,05)$.

FIGURE 1: Content of total extractives of clones' wood in the two evaluated ages. Bars followed by the same lower cases refer to the clone unfold within ages. Capital letters refer to the age unfold within clones. Tukey's test was used $(\alpha=0,05)$.

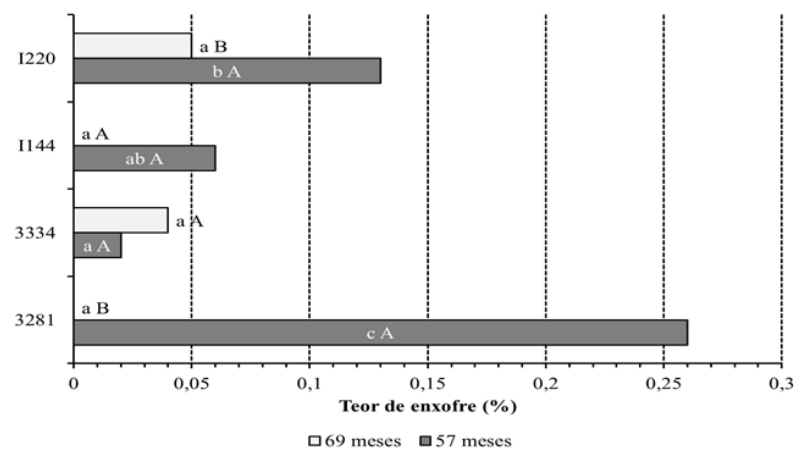

FIGURA 2: Teor de enxofre da madeira dos clones nas duas idades avaliadas. Barras seguidas de mesma letra minúscula referem-se ao desdobramento de clone dentro de idade. Já as letras maiúsculas referem-se ao desdobramento de idade dentro de clone. O teste utilizado foi o de Tukey $(\alpha=0,05)$.

FIGURE 2: Sulfur content of the clones' wood in the two evaluated ages. Bars followed by the same lower cases refer to the clone unfold within ages. Capital letters refer to the age unfold within clones. Tukey's test was used $(\alpha=0,05)$.

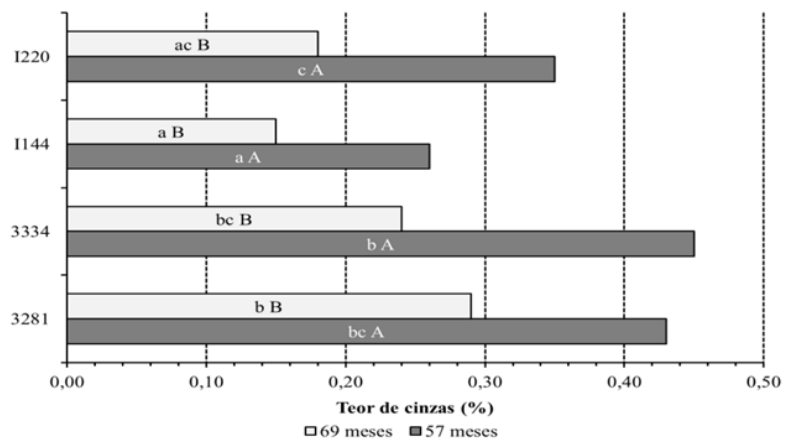

FIGURA 3: Teor de cinzas da madeira dos clones nas duas idades avaliadas. Barras seguidas de mesma letra minúscula referem-se ao desdobramento de clone dentro de idade. Já as letras maiúsculas referem-se ao desdobramento de idade dentro de clone. O teste utilizado foi o de Tukey $(\alpha=0,05)$.

FIGURE 3: Ash content of the clones' wood in the two evaluated ages. Bars followed by the same lower cases refer to the clone unfold within ages. Capital letters refer to the age unfold within clones. Tukey's test was used $(\alpha=0,05)$.

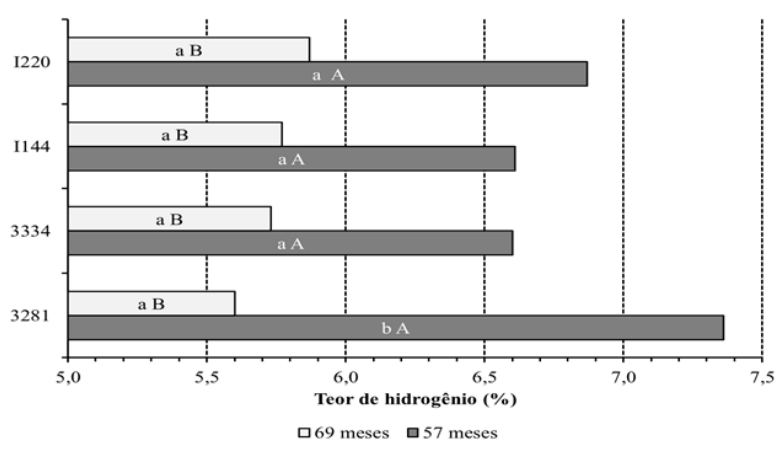

FIGURA 4: Teor de hidrogênio da madeira dos clones nas duas idades avaliadas. Barras seguidas de mesma letra minúscula referem-se ao desdobramento de clone dentro de idade. Já as letras maiúsculas referemse ao desdobramento de idade dentro de clone. O teste utilizado foi o de Tukey $(\alpha=0,05)$.

FIGURE 4: Hydrogen content of the clones' wood in the two evaluated ages. Bars followed by the same lower cases refer to the clone unfold within ages. Capital letters refer to the age unfold within clones. Tukey's test was used $(\alpha=0,05)$. 


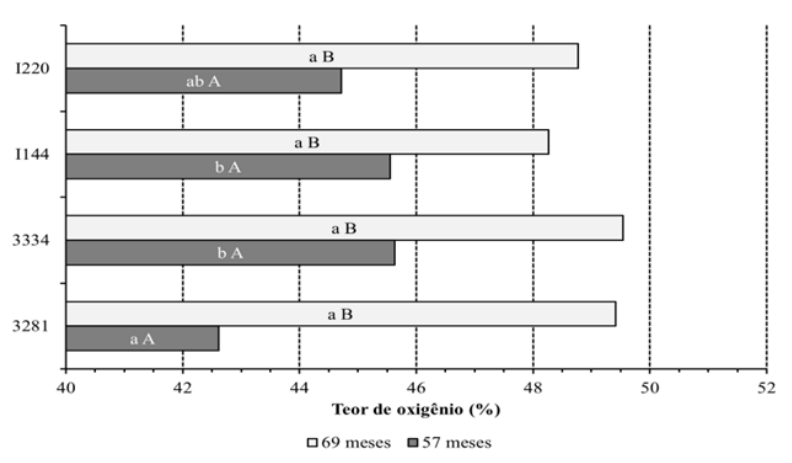

FIGURA 5: Teor de oxigênio da madeira dos clones nas duas idades avaliadas. Barras seguidas de mesma letra minúscula referem-se ao desdobramento de clone dentro de idade. Já as letras maiúsculas referem-se ao desdobramento de idade dentro de clone. O teste utilizado foi o de Tukey $(\alpha=0,05)$.

FIGURE 5: Oxygen content of the clones' wood in the two evaluated ages. Bars followed by the same lower cases refer to the clone unfold within ages. Capital letters refer to the age unfold within clones. Tukey's test was used $(\alpha=0,05)$.

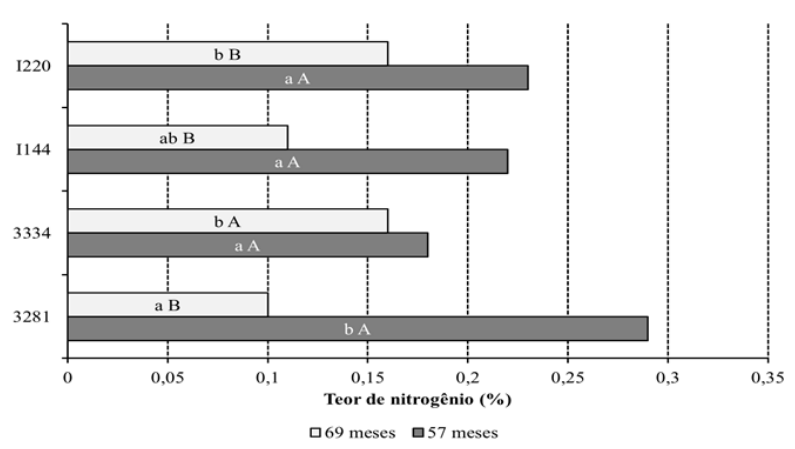

FIGURA 6: Teor de nitrogênio da madeira dos clones nas duas idades avaliadas. Barras seguidas de mesma letra minúscula referem-se ao desdobramento de clone dentro de idade. Já as letras maiúsculas referem-se ao desdobramento de idade dentro de clone. O teste utilizado foi o de Tukey $(\alpha=0,05)$.

FIGURE 6: Nitrogen content of the clones' wood in the two evaluated ages. Bars followed by the same lower case refer to the clone unfold within the age. Capital letters refer to the age unfold within clones. Tukey's test was used $(\alpha=0,05)$. entre esses fatores no PCS da madeira (Tabela 2). Esse resultado evidencia a dependência do valor calórico da interação entre os constituintes químicos, ou a faixa de variação encontrada para a composição química não foi suficiente para expressar diferenças significativas no poder calorífico.

Avaliando o teor de nitrogênio (Figura 6), somente o clone 3334 não apresentou diferença estatística entre as idades de 57 e 69 meses. Para os demais clones pode-se observar um decréscimo desse constituinte elementar com o aumento da idade. Elevados valores de nitrogênio são indesejáveis, pois a liberação desse constituinte na atmosfera pode ocasionar a formação de óxidos tóxicos ( $\mathrm{NOx}$ ), promover a poluição do ar, formação de chuva ácida (DEMIRBAS, 2004; OBERNBERGER et al., 2006) e a diminuição do valor calórico do combustível (HUANG et al., 2009).

As diferenças significativas dos teores de extrativos totais, cinzas, hidrogênio, nitrogênio e oxigênio para todos os clones avaliados em cada idade permitem a seleção de materiais genéticos adequados para a produção de carvão vegetal e/ou bioenergia. Aos 57 meses, o clone 3281 apresentou potencial para a produção de bioenergia, apresentando alto teor de hidrogênio e baixo teor de oxigênio em relação aos demais materiais genéticos avaliados.

Avaliando o efeito do clone dentro de cada nível de idade, observa-se que para o teor de extrativos totais (Figura 1), os valores médios são estatisticamente diferentes entre os clones avaliados nas idades de 57 e 69 meses. Os clones I144 e I220 apresentaram o maior teor de extrativos totais na idade de 57 meses. Já para a idade de 69 meses os clones I144 e 3281 se destacaram.

Verificou-se para os teores de enxofre (Figura 2), hidrogênio (Figura 4) e oxigênio (Figura 5), analisados na idade de 57 meses, que os valores médios dos clones estudados diferem estatisticamente entre si nessa idade. O contrário pode ser observado para a idade de 69 meses. Dentre os materiais genéticos avaliados na idade de 57 meses observa-se que o clone 3281 apresentou estatisticamente o maior valor de hidrogênio e enxofre e o menor valor médio de oxigênio.

Para os teores de cinzas (Figura 3) e nitrogênio (Figura 6) as diferenças encontradas entre os valores médios dos clones são estatisticamente significativas nas idades analisadas. O maior teor de cinzas, na idade de 57 meses, foi observado para o clone 3334. Já para a idade de 69 meses o 
clone 3281 apresentou o maior teor de constituintes minerais. Esse clone também apresentou o maior teor de nitrogênio na idade de 57 meses em relação aos demais materiais genéticos avaliados.

O comportamento de decréscimo dos teores de cinzas, hidrogênio e nitrogênio com o aumento da idade encontrado nesse estudo permite inferir que esse fator deve ser considerado na avaliação de clones de Eucalyptus spp, visando maximizar o rendimento energético da biomassa e a qualidade do carvão vegetal produzido.

De maneira geral, os resultados encontrados para os teores de extrativos totais, cinzas, hidrogênio, nitrogênio, enxofre e oxigênio, estão de acordo com a literatura (TRUGILHO et al., 2001; TRUGILHO et al., 2003; OLIVEIRA et al., 2010; NEVES et al., 2011; PAULA et al., 2011; PROTÁSIO et al., 2011; SANTOS et al., 2011; REIS et al., 2012).

Quanto à densidade básica, com base nos resultados encontrados, pode-se utilizar a madeira dos clones avaliados na idade de 57 meses para a produção de carvão vegetal, sem comprometer significativamente a densidade relativa aparente do carvão, reduzindo assim o ciclo de corte das árvores, uma vez que não foi observado efeito da idade nessa característica (Tabela 3 e Figura 7). Contudo, devemse considerar as demais características da madeira, especialmente o teor de lignina total (Figura 8).

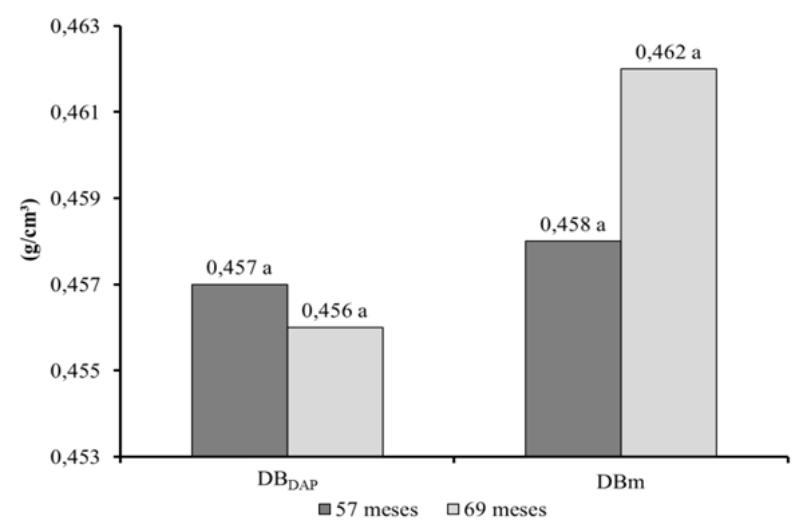

Médias seguidas pela mesma letra não diferem entre si a 5\% de significância pelo Teste $\mathrm{F}$.

FIGURA 7: Efeito da idade na densidade básica da madeira.

FIGURE 7: Effect of age on wood basic density.

Queiroz et al. (2004) verificaram densidade básica média igual a $0,447 \mathrm{~g} / \mathrm{cm}^{3}$ para cavacos da madeira de Eucalyptus grandis e Eucalyptus urophylla aos 6 anos de idade, enquanto Arango
Alzate et al. (2005) encontraram o valor médio de densidade básica equivalente a $0,490 \mathrm{~g} / \mathrm{cm}^{3}$ aos 8 anos de idade para diferentes clones de Eucalyptus spp, corroborando com o observado nesse trabalho.

$\mathrm{O}$ teor de holocelulose apresentou tendência de decréscimo com o aumento da idade, sendo o contrário observado para o teor de lignina total (Figura 8). Isso pode ser considerado uma característica positiva para a utilização bioenergética dos clones de Eucalyptus spp avaliados, visto que a celulose e as hemiceluloses apresentam um perfil instável e pouco resistente diante da degradação térmica da madeira (SANTOS et al., 2011).

Já a lignina é um elemento químico complexo e mais estável termicamente, sendo importante para maximizar o rendimento gravimétrico em carvão vegetal (PROTÁSIO et al., 2012). Logo, o aumento do teor de lignina total com a idade (Figura 8) pode influenciar de maneira considerável a qualidade e produção do carvão vegetal, corroborando com o observado por Trugilho et al. (1997) e Protásio et al. (2012).

Oliveira et al. (2010) encontraram valores médios de holocelulose e lignina total equivalentes a $65,97 \%$ e $29,50 \%$, respectivamente. Trugilho et al. (2003) encontraram para as espécies Eucalyptus grandis e Eucalyptus saligna valores médios $61,52 \%$ e $31,77 \%$ para holocelulose e lignina, diferindo do observado nesse trabalho. A espécie e idade do material genético podem ser consideradas as causas dessa variação.

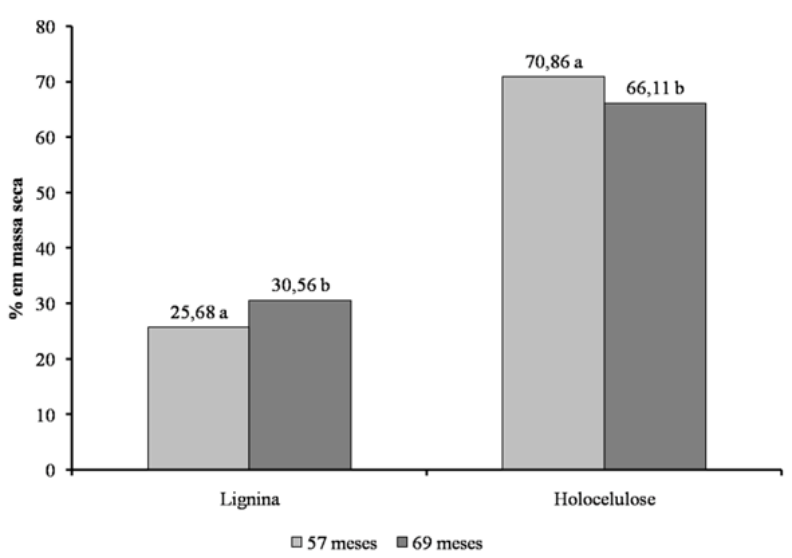

Médias seguidas pela mesma letra não diferem entre si a $5 \%$ de significância pelo Teste $\mathrm{F}$.

FIGURA 8: Efeito da idade nos teores de lignina total e holocelulose na madeira.

FIGURE 8: Effect of age on wood total lignin and holocellulose contents. 
TABELA 4: Efeito de clone nas características da madeira.

TABLE 4: Effect of clone on wood characteristics.

\begin{tabular}{ccccccc}
\hline & \multicolumn{5}{c}{ Características da madeira } \\
\cline { 2 - 7 } Clone & $\mathrm{DB}_{\mathrm{DAP}}\left(\mathrm{g} / \mathrm{cm}^{3}\right)$ & $\mathrm{DBm}\left(\mathrm{g} / \mathrm{cm}^{3}\right)$ & $\begin{array}{c}\text { Lignina } \\
(\%)\end{array}$ & PCS $(\mathrm{kcal} / \mathrm{kg})$ & $\begin{array}{c}\text { Holocelulose } \\
(\%)\end{array}$ & $\begin{array}{c}\text { Carbono } \\
(\%)\end{array}$ \\
\hline 3281 & $0,397 \mathrm{a}$ & $0,406 \mathrm{a}$ & $29,16 \mathrm{a}$ & 4569 & $67,26 \mathrm{a}$ & 46,83 \\
3334 & $0,450 \mathrm{~b}$ & $0,448 \mathrm{~b}$ & $27,48 \mathrm{ab}$ & 4531 & $70,02 \mathrm{~b}$ & 46,03 \\
$\mathrm{I} 144$ & $0,470 \mathrm{~b}$ & $0,476 \mathrm{c}$ & $29,18 \mathrm{a}$ & 4514 & $66,88 \mathrm{a}$ & 46,68 \\
I220 & $0,502 \mathrm{c}$ & $0,504 \mathrm{~d}$ & $27,09 \mathrm{~b}$ & 4572 & $69,34 \mathrm{~b}$ & 46,57 \\
Média & 0,455 & 0,459 & 28,23 & 4547 & 68,38 & 46,53 \\
\hline
\end{tabular}

$\overline{\mathrm{DB}}_{\mathrm{DAP}}=$ densidade básica do diâmetro a altura do peito; $\mathrm{DBm}=$ densidade básica média; $\mathrm{PCS}$ = poder calorífico superior. Médias seguidas pela mesma letra não diferem entre si a 5\% de significância pelo Teste de Tukey.

Estatisticamente, não foi encontrada diferença entre o poder calorífico superior da madeira dos clones de Eucalyptus spp nas idades de 57 e 69 meses, sendo o oposto observado para o teor de carbono na madeira (Figura 9), conforme relatado anteriormente (Tabela 2).

Observa-se o decréscimo do teor de carbono com a idade. Isso não é desejável, uma vez que pode acarretar uma diminuição do poder calorífico, conforme observado por Protásio et al. (2011). Os autores observaram que a cada $1 \%$ de aumento do teor de carbono ocorre um aumento de $64,14 \mathrm{kcal} \mathrm{kg}^{-1}$ no poder calorífico de combustíveis de biomassa. Contudo, essas tendências não foram confirmadas neste trabalho. Isso ocorreu provavelmente devido às pequenas variações

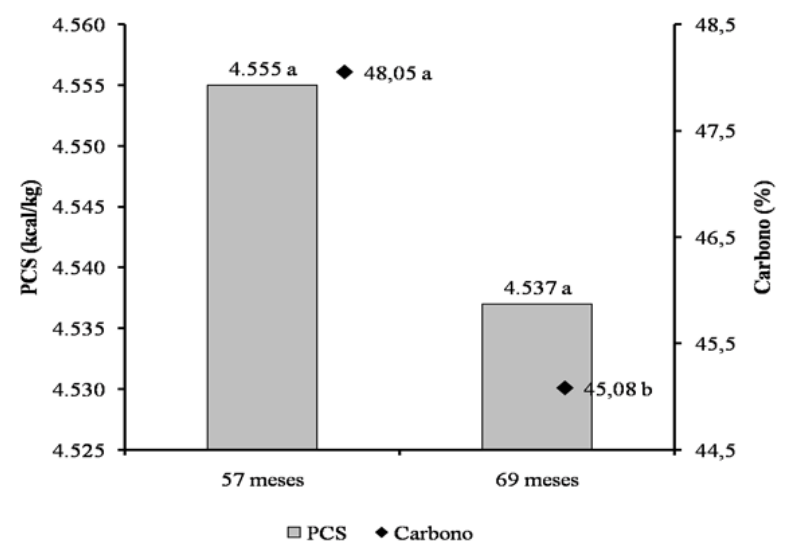

Médias seguidas pela mesma letra não diferem entre si a $5 \%$ de significância pelo Teste $\mathrm{F}$.

FIGURA 9: Efeito da idade no poder calorífico superior (PCS) e no teor de carbono.

FIGURE 9: Effect of age on higher heating value (HHV) and carbon content. observadas entre os constituintes químicos da madeira.

Santos et al. (2011) observaram poder calorífico superior médio variando de $4.274 \mathrm{a} 4.585 \mathrm{kcal}$, para a madeira de diferentes clones de eucalipto aos 7 anos de idade, assemelhando-se ao encontrado para os clones de Eucalyptus spp nas idades de 57 e 69 meses.

Analisando o efeito de clone nas variáveis estudadas, observa-se que os valores de densidade básica média e a obtida no DAP e os teores de lignina e holocelulose são estatisticamente diferentes entre os clones considerados (Tabela 4). Isso resultado propicia a seleção dos melhores clones para a produção de carvão vegetal.

O clone I220 apresentou maiores valores de densidade básica média e no DAP (Tabela 4), o que pode proporcionar melhor desempenho desse clone frente à carbonização (TRUGILHO et al., 2001; OLIVEIRA et al., 2010; SANTOS et al., 2011; PROTÁSIO et al., 2012). Além disso, quanto maior a densidade básica da madeira maior a densidade relativa aparente do carvão vegetal (BRITO; BARRICHELO, 1980; VALE et al., 2001; VALE et al., 2010) e maior a resistência do carvão à abrasão nos altos fornos siderúrgicos.

Demaneira geral, oteor de lignina apresentou pouca variação entre os clones analisados. Os clones I144 e 3281 se destacaram por apresentarem os maiores valores médios de lignina total e os menores valores médios de holocelulose. Entretanto, esses clones não apresentaram os maiores valores para a densidade básica da madeira. Os valores médios do poder calorífico superior e do teor de carbono da madeira não apresentaram diferenças estatísticas significativas entre os clones avaliados (Tabelas 2 e 4). Esses resultados demonstram a importância 
de considerar a produtividade de lignina, carbono e energia por unidade de volume da madeira, ou seja, multiplicando-se a densidade básica por essas características.

\section{Análise estatística multivariada: componentes} principais

Observa-se que as duas primeiras componentes principais explicaram aproximadamente $78 \%$ da variância dos dados originais (Figura 10). Analisando-se a variância explicada acumulada (Figura 10) observa-se que é possível considerar apenas 2 ou 3 componentes principais, pois a partir desse valor não há grandes alterações nos valores de suas variâncias. Entretanto, optou-se por considerar apenas duas componentes principais devido à grande parcela da variância total dos dados explicada por elas, ou seja, as informações mais relevantes dos dados amostrais originais estão contidas nas duas primeiras componentes principais.

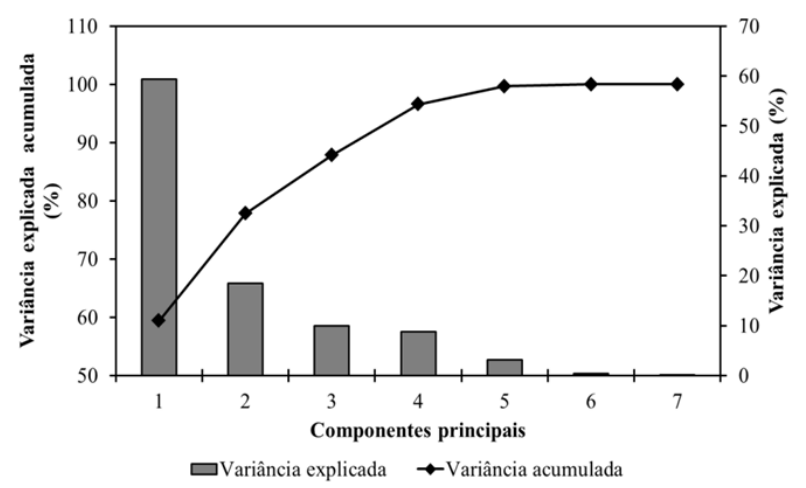

FIGURA 10: Variância explicada acumulada e autovalores das sete primeiras componentes principais.

FIGURE 10: Variance explained cumulative and eigenvalues of the seven first main components.

Na componente principal 1 a densidade básica média e no DAP, o poder calorífico superior e o teor de extrativos totais apresentaram, em módulo, baixos autovetores (Tabela 5). Esse resultado é um indicativo da correlação entre as características químicas da madeira e pode influenciar de forma significativa a qualidade do carvão vegetal e a seleção de clones de Eucalyptus para a produção de bioenergia.

Para a componente principal 2 observa-se que a densidadebásica média e no DAP apresentaram, em módulo, os maiores autovetores (Tabela 5). Analisando-se os sinais do autovetores pode-se constatar que quanto menores os escores dessa componente principal, mais propício será o clone de Eucalyptus spp para o uso bioenergético, pois maior será a sua densidade básica média. Observa-se ainda a correlação positiva entre a densidade básica média e a obtida no DAP. Esse resultado é um indicativo de que a posição de amostragem no DAP pode ser utilizada para a determinação da densidade básica média da árvore e seleção de clones de Eucalyptus spp visando ao uso bioenergético.

TABELA 5: Autovetores normalizados das componentes principais 1 e 2 .

TABLE 5: $\quad$ Normalized eigenvectors of main components 1 and 2.

\begin{tabular}{|c|c|c|}
\hline Variáveis originais & $\begin{array}{l}\text { Componente } \\
\text { principal } 1\end{array}$ & $\begin{array}{l}\text { Componente } \\
\text { principal } 2\end{array}$ \\
\hline $\begin{array}{l}\text { Densidade básica } \\
\text { média }\end{array}$ & 0,108 & $-0,639$ \\
\hline $\begin{array}{c}\text { Densidade básica } \\
\text { no DAP }\end{array}$ & 0,091 & $-0,644$ \\
\hline $\begin{array}{l}\text { Poder calorífico } \\
\text { superior }\end{array}$ & $-0,189$ & $-0,008$ \\
\hline Extrativos totais & 0,118 & $-0,192$ \\
\hline Lignina & 0,335 & 0,214 \\
\hline Cinzas & $-0,306$ & 0,218 \\
\hline Holocelulose & $-0,333$ & $-0,158$ \\
\hline Carbono & $-0,347$ & $-0,057$ \\
\hline Hidrogênio & $-0,366$ & $-0,075$ \\
\hline Nitrogênio & $-0,357$ & $-0,091$ \\
\hline Enxofre & $-0,323$ & 0,032 \\
\hline Oxigênio & 0,356 & 0,060 \\
\hline $\begin{array}{c}\text { Variância } \\
\text { Explicada (\%) }\end{array}$ & 59,4 & 18,5 \\
\hline $\begin{array}{c}\text { Variância } \\
\text { Acumulada (\%) }\end{array}$ & 59,4 & 77,9 \\
\hline Autovalores & 7,13 & 2,21 \\
\hline
\end{tabular}

Analisando-se a distribuição gráfica dos tratamentos considerados (Tabela 1) em relação às duas primeiras componentes principais podese perceber a distinta separação entre os clones de idades diferentes (Figura 11). Esse resultado indica, de maneira geral, o significativo efeito da idade na qualidade da madeira dos clones de Eucalyptus spp avaliados visando à produção de carvão vegetal 


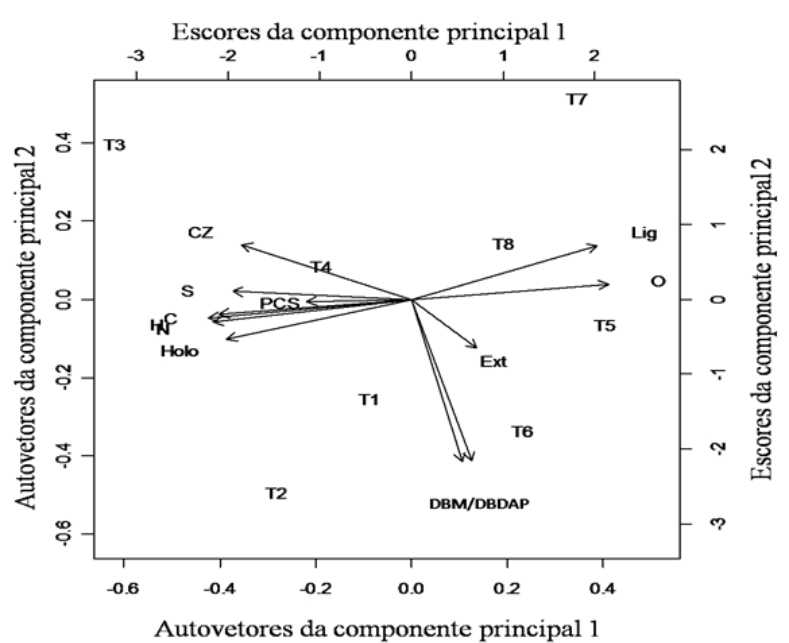

Em que: $\mathrm{CZ}=$ cinzas; $\mathrm{C}=$ carbono; $\mathrm{H}=$ hidrogênio; $\mathrm{N}=$ nitrogênio; $\mathrm{S}=$ enxofre; Holo = holocelulose; $\mathrm{PCS}=$ poder calorífico superior; $\mathrm{DBM}=$ densidade básica média; DBDAP = densidade básica no DAP; Ext $=$ extrativos totais; $\mathrm{Lig}=$ lignina total; T1, T2, T3, T4, T5, T6, T7 e T8 = tratamentos considerados.

FIGURA 11: Diagrama de ordenação dos tratamentos considerando os escores e autovetores das componentes principais 1 e 2 .

FIGURE 11: Ordination diagram of the treatments considering the scores and eigenvectors of the main components 1 and 2 .

e/ou bioenergia.

Observa-se que os clones de Eucalyptus spp com a idade de 69 meses apresentaram os maiores valores de lignina, mas não os maiores valores para a densidade básica da madeira (Figura 11). Para essa característica, os clones I144 e I220 de 57 meses se destacaram. Os clones de 69 meses de idade apresentaram os menores valores para os teores de holocelulose, cinzas, carbono, hidrogênio, nitrogênio, enxofre e para o poder calorífico superior. O oposto foi observado para os clones de 57 meses de idade.

\section{CONCLUSÕES}

Os clones avaliados apresentaram potencial para a produção de carvão vegetal considerando-se as características avaliadas na madeira.

Observou-se, de maneira geral, efeito da idade na qualidade da madeira. Os teores de cinzas, hidrogênio e nitrogênio tenderam a diminuir com o aumento da idade para todos os clones avaliados. Já o teor de oxigênio apresentou um acréscimo com a idade dos clones. O teor de lignina apresentou significativo aumento com a idade, sendo o oposto observado para o teor de holocelulose.

Os clones de 69 meses apresentaram os maiores teores de lignina, mas não os maiores valores para a densidade básica da madeira. Já os clones I144 e I220 apresentaram os maiores valores médios para essa característica, o que pode ser benéfico para a produção de carvão vegetal.

\section{AGRADECIMENTOS}

Os autores expressam seus sinceros agradecimentos ao CNPq e Fapemig pelo auxílio financeiro, à Saint-Gobain Pam Bioenergia pela doação do material biológico utilizado e à equipe do Laboratório Multiusuário de Biomateriais (Departamento de Ciências Florestais) da Universidade Federal de Lavras pelo auxílio na realização dos ensaios.

\section{REFERÊNCIAS BILIOGRÁFICAS}

ABRAF. Anuário estatístico da ABRAF 2012: ano base 2011. Brasília, DF: ABRAF, 2012. 150 p. ARANGO ALZATE, S. B. et al. Variação longitudinal da densidade básica da madeira de clones de Eucalyptus grandis Hill ex Maiden, E. saligna $\mathrm{Sm}$. e E. grandis $x$ urophylla. Scientia Forestalis, Piracicaba, SP, n. 68, p. 87-95, 2005.

ASSIS, M. R. et al. Qualidade e rendimentos do carvão vegetal de um clone híbrido de Eucalyptus grandis $\mathrm{x}$ Eucalyptus urophylla. Pesquisa Florestal Brasileira, Colombo, PR, v. 32, n. 71, p. 291-302, 2012. DOI: $10.4336 / 2012 . p f b .32 .71 .291$

ASSOCIAÇÃO BRASILEIRA DE NORMAS TÉCNICAS - ABNT. NBR 11941: Madeira determinação da densidade básica. Rio de Janeiro: 2003. 6p.

ASSOCIAÇÃO BRASILEIRA DE NORMAS TÉCNICAS. NBR 8633: Carvão vegetal determinação do poder calorífico. Rio de Janeiro, $1984.13 \mathrm{p}$.

ASSOCIAÇÃO BRASILEIRA TÉCNICA DE CELULOSE E PAPEL. M-11/77: Teor de cinzas. São Paulo, Brasil, 1974a. 8 p.

ASSOCIAÇÃO BRASILEIRA TÉCNICA DE CELULOSE E PAPEL. M3/69: Métodos de ensaio. São Paulo, Brasil, 1974b. 8 p.

BARCELLOS, D. C. et al. O estado da arte 
da qualidade da madeira de eucalipto para a produção de energia: um enfoque nos tratamentos silviculturais. Biomassa e Energia, Viçosa, MG, v. 22, n. 1, p.141-158, 2005.

BECH, N.; JENSEN, P. A.; DAM-JOHANSEN, $\mathrm{K}$. Determining the elemental composition of fuels by bomb calorimetry and the inverse correlation of HHV with elemental composition. Biomass and Bioenergy, Amsterdam, v. 33, n. 3, p. 534-537, 2009.

BILGEN, S.; KAYGUSUZ, K. The calculation of the chemical exergies of coal-based fuels by using the higher heating values. Applied Energy, London, v. 85, n. 8, p. 776-785, 2008.

BRAND, M. A. Energia de biomassa florestal. Rio de Janeiro: Interciência, 2010. 131 p.

BRITO, J. O.; BARRICHELO, L. E. G. Correlações entre características físicas e químicas da madeira e a produção de carvão: II. densidade da madeira X densidade do carvão. IPEF, Piracicaba, SP, n. 20, p. 101-113, 1980.

DEMIRBAS, A. Combustion characteristics of different biomass fuels. Progress in Energy and Combustion Science, Pittsburgh, v. 30, n. 2, p. 219-230, 2004.

DOWNES, G. M. et al. Sampling plantation eucalypts for wood and fibre properties. Collingwood, Vic.: CSIRO Australia, 1997. 126 p.

EMPRESA DE PESQUISA ENERGÉTICA. Projeção da demanda de energia elétrica para os próximos 10 anos (2011 - 2020). Rio de Janeiro, RJ, MME, 2011. 105 p. Série Estudos de Energia. Nota técnica DEA 03/11.

FERREIRA, D. F. Estatística Multivariada. 1. ed. Lavras: UFLA, $2008 \mathrm{p}$.

GOLDSCHIMID, O. Ultraviolet spectra. In: SARKANEN, K. V.; LUDWIG, C. H. Lignins: occurrence, formation, structure and reactions. New York: John Wiley \& Sons, 1971. p. 241-266.

GOMIDE, J. L.; DEMUNER, B. J. Determinação do teor de lignina em material lenhoso: método Klason modificado. O Papel, São Paulo, v. 47, n. 8, p. 36-38, 1986.

HUANG, C.; HAN, L.; YANG, Z.; LIU, X. Ultimate analysis and heating value prediction of straw by near infrared spectroscopy. Waste Management, Oxford, v. 29, n. 6, p. 1793-1797, 2009.

HUSSON, F; LE, S.; CADORET, M. SensoMineR: Sensory data analysis with R. R package version 1.15. 2012. Disponível em: http://cran.rproject.org/web/packagesSensoMineR/ index.html. Acesso em: 14 out. 2012.
MINGOTI, S. A. Análise de dados através de métodos de estatística multivariada: uma abordagem aplicada. Belo Horizonte: UFMG, 2005. $297 \mathrm{p}$.

NEVES, T. A. et al. Avaliação de clones de Eucalyptus em diferentes locais visando à produção de carvão vegetal. Pesquisa Florestal Brasileira, Colombo, v. 31 , n. 68 , p. 319-330, 2011. DOI: 10.4336/2011.pfb.31.68.319

OBERNBERGER, I.; BRUNNER, T.; BARNTHALER, G. Chemical properties of solid biofuels-significance and impact. Biomass and Bioenergy, Amsterdam, v. 30, n. 11, p. 973-982, 2006.

OLIVEIRA, A. C. et al. Parâmetros de qualidade da madeira e do carvão vegetal de Eucalyptus pellita F. Muell. Scientia Forestalis, Piracicaba, SP, v. 38, n. 87, p. 431-439, 2010.

PAULA, L. E. R. et al. Characterization of residues from plant biomass for use in energy generation. Cerne, Lavras, MG, v. 17, n. 2, p. 237-246, 2011.

PINHEIRO, P. C. C. et al. A produção de carvão vegetal. Belo Horizonte, 2006. 103 p.

PROTÁSIO, T. P. et al. Relação entre o poder calorífico superior e os componentes elementares e minerais da biomassa vegetal. Pesquisa Florestal Brasileira, Colombo, PR, v. 31, n. 66, p. $122-133$, 2011. DOI: 10.4336/2011.pfb.31.66.113

PROTÁSIO, T. P. et al. Análise de correlação canônica entre características da madeira e do carvão vegetal de Eucalyptus. Scientia Forestalis, Piracicaba, SP, v. 40, n. 95, p. 317-326, 2012.

QUEIROZ, S. C. S. et al. Influência da densidade basica da madeira na qualidade da polpa kraft de clones hibridos de Eucalyptus grandis W. Hill ex Maiden x Eucalyptus urophylla S. T. Blake. Revista Árvore, Viçosa, MG, v. 28, n. 6, p. 901-909, 2004. R DEVELOPMENT CORE TEAM. R: a language and environment for statistical computing. Vienna, Austria: R Foundation for Statistical Computing, 2012. R version 2.15.1. Disponível em: $<\mathrm{http}: / /$ www.R-project.org>. Acesso em: 14 out. 2012.

REIS, A. A. et al. Composição da madeira e do carvão vegetal de Eucalyptus urophylla em diferentes locais de plantio. Pesquisa Florestal Brasileira, Colombo, PR, v. 32, n. 71, p. 277-290, 2012. DOI: 10.4336/2012.pfb.32.71.277

SANTOS, R. C. et al. Correlações entre os parâmetros de qualidade da madeira e do carvão vegetal de clones de eucalipto. Scientia Forestalis, Piracicaba, SP, v. 39, n. 90, p. 221-230, 2011. SEVERO, E. T. D.; CALONEGO, F. W.; 
SANSÍGOLO, C. A. Composição química da madeira de Eucalyptus citriodora em função das direções estruturais. Silva Lusitana, Lisboa, Portugal, v. 14, p. 113-126, 2006.

TRUGILHO, P. F. Densidade básica e estimativa de massa seca e de lignina na madeira em espécies de Eucalyptus. Ciência e Agrotecnologia, Lavras, MG, v. 33, n. 5, p. 1228-1239, 2009.

TRUGILHO, P. F.; LIMA, J. T.;MORI, F. A. Correlação canônica das características químicas e físicas da madeira de clones de Eucalyptus grandis e Eucalyptus saligna. Cerne, Lavras, MG, v. 9, n. 1, p. 066-080, 2003.

TRUGILHO, P. F. et al. Aplicação da análise de correlação canônica na identificação de índices de qualidade da madeira de eucalipto para a produção de carvão vegetal. Revista Árvore, Viçosa, MG, v. 21, n. 2 , p. $259-267,1997$.

TRUGILHO, P. F. et al. Avaliação de clones de Eucalyptus para a produção de carvão vegetal. Cerne, Lavras, MG, v. 7, n. 2, p. $104-114,2001$. VALE, A. T.; DIAS, I. S.; SANTANA, M. A. E. Relações entre propriedades químicas, físicas e energéticas da madeira de cinco espécies de cerrado. Ciência Florestal, Santa Maria, RS, v. 20, n. 1, p. 137-145, 2010.

VALE, A. T. et al. Relações entre a densidade básica da madeira, o rendimento e a qualidade do carvão vegetal de espécies do cerrado. Revista Árvore, Viçosa, MG, v. 25, n. 89, p. 89-95, 2001. 